\title{
CARTOX Grade 1
}

National Cancer Institute

\section{Source}

National Cancer Institute. CART OX Grade 1. NCI Thesaurus. Code C155296.

A score of 7-9 on the CART OX-10 assessment, indicating mild neurotoxicity. 